\title{
Proteomics of the human malaria parasite Plasmodium falciparum
}

\author{
Paul FG Sims, PhD \\ University of Manchester, Faculty of Life Sciences, Jackson's Mill, PO Box 88, Manchester, M60 \\ 1QD, UK Tel.: +44 1613064171 Fax: +44 1612360409 paul.sims@manchester.ac.uk
}

John E Hyde, DPhil ${ }^{\dagger}$

University of Manchester, Faculty of Life Sciences, Jackson's Mill, PO Box 88, Manchester, M60 1QD, UK Tel.: +44 1613064185 Fax: +44 1612360409 john.hyde@manchester.ac.uk

\begin{abstract}
The lethal species of malaria parasite, Plasmodium falciparum, continues to exact a huge toll of mortality and morbidity, particularly in sub-Saharan Africa. Completion of the genome sequence of this organism and advances in proteomics and mass spectrometry have opened up unprecedented opportunities for understanding the complex biology of this parasite and how it responds to drug challenge and other interventions. This review describes recent progress that has been made in applying proteomics technology to this important pathogen and provides a look forward to likely future developments.
\end{abstract}

\section{Keywords}

cell cycle; drug challenge; mass spectrometry; metabolic labeling; post-translational modifications; quantitative proteomics; subproteome analysis

The malaria parasite Plasmodium falciparum is one of four species of Plasmodium in the phylum Apicomplexa that infect humans, and is responsible for virtually all deaths from this disease as well as high levels of morbidity. Malaria has a profound impact on the public health and education systems, economies and social structures of many developing countries, particularly in Africa. Proteomic analysis of this deadly parasite began nearly two decades ago with investigations of 2D gel patterns of parasite proteins before and after application of the antimalarial drug mefloquine [1], and as an indicator of natural genetic variation in parasite populations [2]. Such studies were limited at that time by the inability to easily identify proteins of interest on 2D gels, a situation that has dramatically changed in recent years with the completion of the genome sequencing project for $P$. falciparum [3], and the rapid advances made in mass spectrometry (MS) techniques as applied to proteins [4]. Considerable progress has also been made in the analysis of 2D gel patterns from the related apicomplexan pathogen Toxoplasma gondii [5], whose genome sequence is almost complete but whose coding sequences are interrupted by introns at a much higher frequency than those of $P$. falciparum and other Plasmodium species, and thus pose greater problems for interpretation of mass spectrometric data.

${ }^{\dagger}$ Author for correspondence University of Manchester, Faculty of Life Sciences, Jackson's Mill, PO Box 88, Manchester, M60 1QD, UK Tel.: +44 1613064185 Fax: +44 1612360409 john.hyde@ manchester.ac.uk. 


\section{The malarial proteome across the cell cycle}

Although $P$. falciparum is only single celled, this eukaryotic organism undergoes dramatic morphological changes, both in shape and size, as it passes through the various stages of its complex life cycle in two hosts; the intermediate human host, and the definitive mosquito host of the Anopheles genus, where sexual reproduction occurs. These stages include:

- Invasion by the ookinete of the female mosquito mid-gut after the obligatory blood meal from the human target

- Transport of the sporozoite to the salivary glands

- Invasion of the human liver by sporozoites after the infected mosquito bites

- Invasion of the erythrocytes by merozoites released from the liver

- Cyclical reinvasion of erythrocytes by blood-form merozoites

- Differentiation of asexual to presexual stages (gametocytes) in the erythrocytes and to mature sexual forms (gametes) in the mosquito

- as well as the growth stages within these various tissues.

As the parasite proceeds through these numerous stages, the profiles of protein expression change dramatically, particularly with respect to antigens on the outer membrane of the invasive forms, and proteins that the parasite transports to the surface of the infected host cells.

As the genome sequence neared completion, two groups in particular undertook large-scale analysis of proteins extracted from some of the more experimentally accessible stages of the $P$. falciparum life cycle, primarily to catalog the changes in the protein repertoire that occur [6,7]. Multidimensional protein identification technology (MudPIT) involves the use of 2D nanoflow high-performance (HP) liquid chromatography (LC) directly coupled to electrospray ionization (ESI) MS to fractionate and analyze parasite extracts. In this way, Florens and coworkers were able to identify in the parasite line 3D7 just over 2400 proteins from the asexual sporozoite, trophozoite and merozoite stages, and from gametocytes as representative of sexual stage parasites, sorting them into ten functional categories [6]. The remainder, hypothetical and unknown proteins, made up by far the largest category at each stage. Although, in general, genes expressing proteins with related functions are distributed apparently randomly among the parasite's 14 chromosomes, a number of clustered genes were identified that encode coexpressed proteins, suggesting one possible mechanism for the control of gene expression, an aspect of the parasite's biology about which relatively little is known. Thus, 98 clusters containing three loci were identified, 32 clusters containing four loci, five clusters containing five loci and three clusters containing six loci. Unexpectedly, the antigenically variant proteins of the var and rif genes, originally characterized as important molecules on the surface of infected erythrocytes, were also expressed to a high degree in sporozoites, with over 20 isoforms of each gene product detectable in the mass spectrometer. The authors suggest that this may be a part of the survival strategy of the nonreplicative sporozoites as they migrate to the liver after inoculation into the bloodstream.

The analysis undertaken by Lasonder and coworkers was similar, albeit of NF54, the parental parasite line of 3D7, rather than 3D7 itself [7]. However, this group adopted a complementary approach to sample classification, dividing the sexual stages into the gametocytes (formed in the human erythrocyte) and gametes (formed in the mosquito gut after the blood meal), while treating the asexual blood stages as just a single group. In this way, they identified 1289 proteins, 714 of which were present in asexual blood stages, 931 in gametocytes and 645 in gametes. Among the last two categories, 575 appeared to be 
unique to the sexual stages and included a subset of proteins containing domains indicative of a role in cell-cell interactions. Interestingly, peptides were also seen in the mass spectra with significant matches to six-frame translation of the parasite genome, but which were not in the protein set predicted by computational methods. This result emphasizes the limitations of the latter and indicates the value of such direct proteomic analyses to sequence database annotation.

Since publication of the above data for $P$. falciparum, a similar approach has been applied to the asexual and sexual stages of the rodent parasite Plasmodium berghei, a more amenable experimental model for the complete life cycle of the parasite than P. falciparum [8]. In addition to classifying the 1836 securely identified $P$. berghei proteins by the stage specificity of their expression, these authors also divided the data set according to whether they were likely to be housekeeping proteins, whether their expression was altered in response to the shift from the mammalian to the insect host, and whether they were involved in strategy-specific expression, (i.e., host cell invasion, asexual multiplication or sexual development). Just over half of the proteins were detected solely in one stage of the life cycle, indicating that a considerable degree of specialization is required at the molecular level to support the demanding program of morphological and metabolic transitions that the parasite undergoes.

\section{Exploring subsections of the proteome}

Whole proteome analysis presents a major challenge. With current and foreseeable technology, the less abundant proteins in such highly complex mixtures are always likely to escape detection. Moreover, very large amounts of data processing are required even at levels of analysis where not all proteins are identified. However, comprehensive proteome determination is often not necessary to obtain useful answers to specific questions, and analysis of less complex mixtures will inevitably result in a more complete description of their protein content. For these reasons, several studies have targeted organellar proteins or other subsections of the malarial proteome. For example, co-immunoprecipitation has been used to enrich for proteins of the Maurer's clefts, which are involved in protein trafficking between the parasitophorous vacuole and the host plasma membrane [9]. Using MudPIT technology for analysis of the immunoprecipitates, a novel subtelomeric gene family encoding 11 transmembrane proteins was identified. Moreover, the authors were able to establish that most of these proteins were expressed and localized to the Maurer's clefts during the trophozoite stage of the parasite's life cycle. Subsequent work used a novel method to distinguish between parasite and host proteins in an infected erythrocyte ghost fraction, identifying 78 parasite proteins, including seven that were not previously associated with Maurer's clefts [10]. In addition, these cleft-associated proteins included the products of six contiguous genes from chromosome 5, thereby providing further evidence that genes encoding some functionally related proteins may be clustered in the parasite genome.

Similar subproteome analyses have been performed on the parasitophorous vacuole itself [11], the rhoptries (critical organelles deployed during merozoite invasion of fresh erythrocytes) $[12,13]$, proteins exported to the surface of infected cells [14], proteins expressed during the ookinete stage of the parasite in the mosquito host [15] and those expressed in either of the male or female gametocytes [16]. In the last study, conducted with $P$. berghei, an elegant method of physically separating the male and female gametocytes by flow cytometry allowed assignment of 305 proteins to the male form, 170 to the female form and only 69 proteins common to both, indicative of a high level of divergence in sexspecific mechanisms. In addition, the identification of both protein kinases and phosphatases associated with one or other of the gametocyte types demonstrated for the first time the existence of sex-specific regulatory pathways [16]. Analysis of the liver stages of $P$. 
falciparum poses a particular challenge as malaria parasites are highly host specific and these stages are not easily accessible either in culture or in vivo. However, their characterization is of major importance to the development of vaccines designed to promote T-lymphocyte responses that can destroy liver cells infected by the parasite shortly after the mosquito bite. As for the mosquito stages, animal models will be important in this regard, particularly the rodent parasite systems where liver stages are easily accessed.

Subproteome analysis need not be confined to organelle or life-cycle stages, but can also be applied to proteins with particular biological functions. Thus, proteins able to bind to ferriprotoporphyrin IX, the product of the parasite's degradation of hemoglobin, have been identified [17]. Included amongst these were parasite-encoded glyceraldehyde-3-phosphate dehydrogenase, glutathione reductase and protein disulfide isomerase. Arguably, some of the most important types of malarial subproteomes are those composed of parasite proteins that interact with a particular inhibitor or class of inhibitors. Thus, protein kinases were isolated from a range of organisms, including $P$. falciparum and $T$. gondii using an affinity chromatography approach with known kinase inhibitors as the bound ligands [18]. In this early example, some proteins were identified by protease digestion followed by chemical microsequencing of separated peptides. Graves and coworkers used extracts of infected and uninfected cells in a similar approach attempting to identify host and parasite proteins that interact with the quinoline inhibitors hydroxychloroquine and primaquine [19]. Although the approach was validated in these experiments by the use of MS to reproducibly identify the human proteins aldehyde dehydrogenase 1 and quinone reductase 2 as selectively interacting with the inhibitors, no parasite proteins were detected. This was probably due to the significant excess of host proteins in the samples analyzed, and begs the question as to whether such an analysis might not be more successful using extracts prepared from purified parasite material. In any case, the determination of further functionally related proteomes should be worthwhile and it is likely that, in the future, the protein targets of novel inhibitors could be directly identified in this way, or at least narrowed down to a small number of candidate molecules. Recently, small synthetic molecules tagged with a fluorophore or radiolabel for visualization and directed towards different families of enzymes, such as proteases or phosphatases, have been developed as activity-based probes. These should also provide a complementary approach to identifying novel inhibitors of the parasite [20] and studying the roles of particular target enzymes by chemical knockout techniques [21,22].

The subproteome consisting of the surface proteins of $P$. falciparum is of particular relevance in the context of malaria vaccine targets. However, this proteome is highly variable, presumably due to host cell immune pressure, and has proved difficult to study using classical nucleic acid-based methods. Nevertheless, a study using tandem MS (MS/ MS) to characterize the variant antigens expressed on the surface of malaria-infected erythrocytes that bind to chondroitin sulfate A (CSA) offers encouragement that even this recalcitrant target may be accessible using proteomic approaches [23]. Four novel variants of $P$. falciparum erythrocyte membrane protein 1 were detected in CSA-binding parasites, a result that is relevant to our understanding of pregnancy-associated malaria, where binding to this ligand has been shown to be important for parasite sequestration in the placenta [24]. Very recently, a more general study that is relevant to the development of blood-stage vaccines, and important for the understanding of erythrocyte invasion in particular, examined the complement of proteins carried on the surface of the infective merozoite [25]. Certain antigens known to play critical roles in invasion or protective immunity, such as merozoite surface protein (MSP)-1, MSP-3 and apical membrane antigen-1, already form the basis for a number of experimental vaccines undergoing clinical trials. To explore this subproteome further, detergent-resistant membranes of late-stage schizonts (i.e., just prior to the release of free merozoites) were isolated and found to be especially enriched in glycosylphosphatidylinositol (GPI)-anchored proteins, multimembranespanning proteins and 
proteins destined for export into the erythrocyte, as well as proteins from rhoptries, organelles that are required for the penetration of host cells. This type of proteomic analysis has allowed a detailed working model of the merozoite surface of the parasite to be produced.

\section{Post-translational modification of the malarial proteome}

In common with studies on many other organisms, MS of $P$. falciparum extracts separated on 2D gels highlights the presence of numerous proteins that are represented by more than one spot in the gel. These isoforms of a particular protein are assumed to be posttranslationally modified variants of that protein [26]. Biochemical studies have indicated that, at the very least, glycosylation [27] and phosphorylation [28,29] occur quite widely on parasite proteins, while acetylation has been observed on the histones [30] and actin [31]. The major glycosylation event in the blood stages appears to be GPI addition at the Cterminus of the protein for anchoring of certain key antigens (such as MSP-1 and -2) to the membranes of the infected erythrocyte [32]. Internal modifications, such as O-glycosylation at Ser and Thr, and N-glycosylation at asparagine residues, are thought to be absent or at very low levels in $P$. falciparum proteins, although their true extent has been the subject of some debate [27,33,34]. Consistent with the biochemical evidence for phosphorylation, comparative sequence analysis has been used to identify a number of putative parasite kinases, some with atypical structural and functional properties relative to their mammalian counterparts, making them potentially attractive drug targets [35].

Given this preliminary evidence of post-translational modification of parasite proteins, the authors sought to examine in more detail what modifications were occurring on some of the more abundant proteins that had been observed on 2D gels, such as actin I, triosephosphate isomerase, 14-3-3 protein and enolase [26]. Ultimately, the aim is to establish both the type and exact amino acid residue upon which the modification(s) reside(s) for any given protein, together with a biological understanding of when, why and to what extent the modifications occur at different stages of the life cycle or in response to different external stimuli. Phosphorylation was investigated first, given its likely high frequency of occurrence, the relative ease with which it should be detectable in the mass spectrometer and the comparative simplicity of the modification itself. However, when operating in the normal positive-ion mode, the signal derived from phosphorylated peptides is suppressed compared with that seen from their unmodified counterparts, a consequence of the additional negative charge carried by the former. Under these circumstances, peptides of interest often become very minor species in quite complex spectra and can be easily overlooked. This issue can be addressed by the routine inclusion of negative-ion analyses in such a study.

In the authors' laboratory, negative-ion experiments have been performed on both matrixassisted laser desorption/ionization (MALDI) time-of-flight (TOF) and ESI ion-trap instruments. In the former case, it is necessary to switch the sample matrix used from saturated 4-hydroxy-a-cinnamic acid in 50\% acetonitrile plus $0.1 \%$ formic acid to saturated sinnapinic acid in 50\% acetonitrile as well as to reverse the instrument polarity. In the case of the ion-trap spectrometer, only the polarity is changed because the chromatography media used are unstable to the alkaline $\mathrm{pH}$ that might be expected to be more useful for the generation of negative ions. In both cases, these simple adjustments dramatically improve the instruments' sensitivity to modified peptides, greatly aiding their identification. However, it should be noted that protocols and software for amino acid sequence determination from negative-ion data are far less well developed than the methods used to extract such information from positive-ion analyses. Therefore, the authors routinely use the negative-ion data to identify peptides of interest. These are then analyzed for amino acid sequence in positive-ion mode using peptide mass information determined at maximum 
sensitivity in the negative-ion scan to identify the corresponding peaks of interest in the equivalent positive-ion spectrum.

During the course of the analyses described before, certain peptides were identified that were clearly modified to give a mass shift consistent with phosphorylation, but whose behavior during collision-induced dissociation (CID) in the ion trap could not be convincingly ascribed to the loss of a phosphate group. Given the very similar incremental mass changes that are predicted for phosphorylation and sulfonation (average mass 79.9799 and $80.0642 \mathrm{Da}$, respectively), the possibility that sulfonation might account for this aberrant behavior was investigated. Such a modification was recently reported in a study that demonstrated, for the first time, that sulfonation could occur on Ser and Thr residues in proteins from several organisms, including $P$. falciparum, as well as on Tyr [36], as had been established some time before [37]. Consistent with the authors' MS data, biochemical experiments confirm that these atypical (and not uncommon) modifications are indeed not the result of phosphorylation. These observations suggest important future opportunities for proteomic analysis that are likely to make a significant contribution to the understanding of the dynamics of the malarial proteome.

\section{Quantitation of protein changes}

In addition to investigating the qualitative changes in protein patterns accompanying development, it is also important to accurately measure comparative levels of protein expression over the cycle. Similarly, perturbations in such levels that are induced by drug challenge, metabolic stress and so on need to be quantified. In particular, drug challenge can result in changes across a broad spectrum of products, as monitored both at the mRNA level [38] and on 2D protein gels, as demonstrated in the latter case for treatment of parasites with the clinical combination of artemether and lumefantrine where, individually, the two drugs induced opposite effects on the expression levels of certain glycolytic enzymes while exerting a similar influence on those of stress-response proteins [39]. In the high-throughput studies described earlier, only semiquantitative data for protein abundance were obtained, based either on the number of peptides identified for any given protein [6] or the proportion of the total ion current that is accounted for by such peptides [7]. Such data are of value provided the limitations of such approaches are respected. However, recent work from the authors' laboratory has resulted in the development of methodology that is specific for $P$. falciparum and can provide fully quantitative data on the comparative abundance of proteins from parasites cultured under different conditions [26].

There have been two main approaches to comparative quantitation of protein levels by MS in other organisms, metabolic labeling of cells in culture with a heavy isotope derivative of a natural precursor [40], or chemical labeling of protein extracts with isotope-coded affinity tagging (ICAT) [41] or isobaric tags (iTRAQ ${ }^{\mathrm{TM}}$ ) [42], after disruption of the cells of interest. All of these methods take advantage of the fact that when different isotopic forms of the same compound are analyzed in a single mass spectrum, the ratios of the peak areas representing labeled or unlabelled species accurately reflect the relative quantities of the two within a mixture. However, a major advantage of metabolic labeling is that experimental and control cultures are immediately mixed in a known ratio and, thus, all downstream processing, from cell lysis, protein extraction, sample work-up to separation on 2D gels or LC, are identical for each, thus eliminating variability in these steps that could affect the calculated ratio of proteins of interest. In addition to their high cost and the need to chemically label extracts after their release from the cell, there are other drawbacks of the chemical labeling methods. The ICAT technique is dependent upon the presence of cysteine residues, which are of relatively low abundance in the $P$. falciparum proteome $(\sim 1.7$ mole $\%)$. Moreover, users must contend with the occurrence of undesirable side reactions in the 
modification of these residues and the need for considerable optimization [43]. Although these deficiencies have been largely addressed with the introduction of the iTRAQ technology, which targets primary amines present on all peptides, chemical labeling with these reagents is necessarily deferred until after sample alkylation and trypsin digestion, further delaying the point at which differently labeled samples can be combined and treated as one.

As a result of the considerations outlined above, the authors have favored metabolic labeling for quantitative analysis of $P$. falciparum. One advantage of this organism compared with other species in this genus, and indeed to many other cell types, is the ease with which the parasite can be maintained in continuous in vitro culture, using human red blood cells as the host and supported by commercially available tissue-culture medium and human serum or serum substitute. The authors took advantage of this in vitro culture system and adapted their approach from the stable isotope labeling by amino acids in cell culture (SILAC) technology [44], employing heavy isoleucine as the label of choice. This was based on a rationale unique to this parasite and its close relatives. In general, $P$. falciparum derives its amino acids from three sources: degradation of the hemoglobin in the host erythrocyte, transport of free amino acids from the host plasma or culture medium (in the case of in vitro studies), and interconversions between certain pairs of amino acids. For optimal labeling, an amino acid was required that: could not be made from a related amino acid; is absent from human hemoglobin, but is taken up efficiently by the parasite from the culture medium; and is relatively abundant in parasite proteins. This last point is important because it is advantageous for as many as possible of the peptides obtained by tryptic digestion to carry at least one labeled residue, thus allowing them to be clearly identified as arising from either the experimental or control protein samples and to permit values for a number of peptides from a particular protein to be averaged.

Only isoleucine, with an abundance of approximately 9.2 mole $\%$ in $P$. falciparum, fulfilled all of the above criteria. Moreover, it is particularly well suited as a label, since $99.9 \%$ of the approximately 5300 annotated proteins predicted from the complete genome sequence carry isoleucine residues, with an average count of 70 per protein. Thus, more than $60 \%$ of all tryptic peptides over $500 \mathrm{Da}$ determined in silico contain at least one isoleucine residue. This ensures that a good coverage of labeled peptides will be seen in the mass spectrum for the vast majority of proteins, thus providing greater reliability and accuracy. A further benefit of heavy isoleucine (commercially available as ${ }^{13} \mathrm{C}_{6}$ Ile or ${ }^{13} \mathrm{C}_{6},{ }^{15} \mathrm{~N}_{1}$ Ile) is that the mass increment over its unlabelled counterpart is 6 or $7 \mathrm{Da}$, thus providing a clear spectral separation between the isotopic envelopes of equivalent but differentially labeled peptides. Application of this method is neither routine nor cheap, as tissue culture medium devoid of isoleucine must be custom made and the cost of the heavy isotope-labeled amino acid is significant. However, other quantitative approaches are also costly and, after a large initial outlay, the reagents needed for metabolic labeling can be used for many rounds of parasite culture on quite a sizeable scale.

Initial application of this technique to $P$. falciparum, largely to establish proof of principle, involved the study of cell cycle changes where divergent patterns of protein and reported transcript levels were observed, which is indicative of modulation at the translational level [26]. Differences in abundance among post-translationally modified variants of phosphoethanolamine $\mathrm{N}$-methyltransferase and actin-I across the cell cycle were also measured, and the technique can be used to compare rates of turnover among proteins of interest. Parasite responses to equipotent doses of the clinical antimalarial inhibitors pyrimethamine and tetracycline were also monitored, and differential effects for a number of proteins unrelated to likely targets of these drugs were observed. Ongoing work has demonstrated the applicability of this approach to quantitation in high-throughput LC/MS/ 
MS experiments. However, currently, it is not straightforward to analyze quantitative data from high-throughput experiments in this way. The analysis of the latter requires further development of software able to identify and quantify signals from equivalent peptides within the very large data sets generated.

\section{Expert commentary \& five-year view}

In traditional proteomics, including studies of malaria parasites, analysis has almost always been performed with mass spectrometers functioning in positive-ion mode. Although the justification for restricting MALDI analyses in this way is less clear cut, it has historically been the case that negative-ion MS/MS, particularly with nanospray ionization, was much more technically demanding compared with the analysis of positive ions. However, recent developments in instrumentation, coupled with new emitter technologies, have largely removed these considerations. Thus, it is now possible to search directly for negative ions with high sensitivity and specificity [45]. Analyses of this type allow the detection of peptides that are rich in negatively charged amino acid residues, or post-translationally modified with phosphate or sulfate moieties, which are often not seen at all in the positive mode. Thus, a combination of negative- and positive-ion data collected from the same sample should significantly improve sequence coverage and database search scores. The recent release (version 2.1) of the MASCOT ${ }^{\circledR}$ database search software has, for the first time, the capacity to utilize negative- as well as positive-ion data and, therefore, the theoretical benefits of combining such data sets should now be within reach.

If the benefits of negative-ion analysis are to be fully realized with nanospray ESI, modifications to the nanoflow HPLC methods, to which it is normally coupled, are also required. The conventionally used silica-based chromatography matrices are not compatible with the high $\mathrm{pH}$ values needed to obtain the maximum yield of negative ions. However, recently described monolithic stationary phases based on poly(styrene/divinylbenzene) can be used at high (as well as low) $\mathrm{pH}$, and have been successfully coupled with nanospray ESI to give fast and sensitive detection of negatively charged peptides [46]. The use of monolithic columns can also dramatically reduce the length of the chromatographic run required to produce acceptable peptide separation. However, in order to benefit from this, it is imperative that the performance of the mass spectrometer can cope with the reduced time available for analysis. Recently introduced ion-trap equipment does demonstrate muchimproved scan cycle times and should be suitable for this task. Moreover, by combining this with the capacity of such instruments to perform electron-transfer dissociation (ETD) as well as (or possibly instead of) low-energy CID for fragmentation in the second mass spectrometer step (MS2), it should be possible to access more complete amino acid sequence information from longer peptides than at present. It should also be noted that ETD has the potential to be particularly useful for the analysis of post-translational modifications [47]. This is because such modifications are retained during ETD-induced peptide backbone cleavage, in a manner similar to that observed for the products of electron capture dissociation in Fourier transform ion cyclotron resonance instruments, and therefore, the sites of modification can be much more easily located than is often the case with CID.

It would be expected that, for malaria, as well as for other experimental systems, investigations incorporating all of these recent developments in the technology supporting proteomics will significantly improve both the quality and quantity of the data acquired. However, without care, this may create problems rather than solving them. Without the development of the necessary software tools to analyze and archive the results obtained, there is a danger that these data may not be used to their full potential. Particularly important requirements are tools supporting the analysis of high-throughput quantitative experiments and those facilitating dissemination of the results obtained. 
The recent developments in malaria proteomics have heavily depended upon the availability of the complete sequence of the parasite genome. It is only by comparing experimentally determined peptide mass and sequence data against the protein complement predicted from the DNA sequence that full use of such data may be made [7]. However, it is important to realize that, while these protein sequence predictions are generally accurate, the majority have not been experimentally confirmed. As the malarial proteomics effort gathers pace, the opportunity that the gathered data offers with respect to confirming or correcting the genome database annotations must not be overlooked. This important task is rarely, if ever, the primary objective of any experiment and, due to this, its value in this respect may not always be realized. It would be unfortunate if, as a community that has gained so much from the genome sequencing effort, we neglected to add what we can to ensure its accuracy and thus its continuing value. However, even with the completed, annotated genome sequence of the 3D7 line, the interpretation of malarial proteomics data is not straightforward. Thus, it is easy to ignore the fact that $P$. falciparum is anything but a homogeneous organism. It is known that, across the parasite population (estimated in humans alone to number between $10^{16}$ and $10^{18}$ individual parasites worldwide at any given time [48]), there is a high degree of polymorphism affecting many of the antigens presented to the host immune systems. It has also been demonstrated that parasites from different parts of the world vary markedly in the speed at which they adapt genetically to novel drug challenge [49]. There are also likely to be subtle differences in metabolism among strains that are currently completely undiscovered. To address these issues at the genomic level, other cloned laboratory lines of the parasite are now being sequenced to complement the original 3D7 project, as is the genome of a field isolate that has not been subject to possible in vitro genetic modification. This can occur as a result of long periods of laboratory cultivation, and thus, absence of contact for many generations with either human or mosquito host, other than the washed erythrocytes provided in culture. Such extended DNA sequence databases should facilitate improved proteomic analysis in the future.

Of course, proteomics examines only one aspect of cellular function and activity; protein expression. Although this is arguably the most important viewpoint from which to approach the totality of cell biology, ultimately, proteomics data must be integrated with transcriptome data from the same gene set(s) of interest, and with metabolomics data for those proteins involved in the processing of small molecules [50]. Initial attempts at integration of transcriptome and proteome data have been undertaken for both $P$. berghei [8] and $P$. falciparum [51]. In the latter case, mRNA levels were measured on high-density microarrays and the abundance of their cognate proteins estimated semi-quantitatively by the number of MS2 spectra identified per protein. Although there was a moderately high correlation between mRNA and protein levels for each of the life-cycle stages examined, significant discrepancies were observed. These were most often manifest as a delay between the peaks of mRNA transcription and protein amounts, a phenomenon most marked in the gametocyte stages, but also observed for other stages. Thus, for example, transcriptional activity in the merozoite correlated best with the proteome of the ring stage in the following cycle. These observations have general implications for the interpretation of transcriptome data, as well as providing important biological insights into the likely importance in this parasite of post-transcriptional control mechanisms that affect the stability of mRNA species, and the timing and control of their translation.

The scope and utility of these types of approach can also be usefully expanded by comparative genomic analysis in silico across different species of Plasmodium, which, among other things, can reveal rapidly or slowly evolving gene families and conserved elements within noncoding regions that might be of functional significance [8]. Similarly, bioinformatics analyses of the complete genome sequence of $P$. falciparum have led to predictions of large families of parasite proteins with a common property, such as the 
permeome of at least 109 proteins putatively involved in membrane transport [52], and the secretome of some 300-400 members that are synthesized by the parasite and then exported to the host erythrocyte $[53,54]$. Proteomic analyses will be important in establishing the validity and completeness of these predicted protein subgroups, as well as their biological significance in the different stages of the life cycle.

Proteomic and MS methodologies are also being exploited with more direct clinical applications in mind. Thus, the hemozoin pigment produced by the parasite as a waste product of hemoglobin digestion (the insoluble sequestered form of ferriprotoporphyrin IX) can be detected in infected blood samples by laser desorption MS with a greater sensitivity than can be achieved by conventional screening of blood slides in the light microscope; an important consideration when rapid diagnosis can make the difference between survival or death [55]. MALDI-TOF analysis has also been applied to DNA samples from parasites that differ in their susceptibility to the clinical antifolate drugs, as a potential basis for a highthroughput method of detecting the well-characterized point mutations in the target genes that underlie the drug resistance phenotype [56]. These novel applications, together with the identification of potential drug targets by ligand binding to parasite extracts (mentioned earlier), are indicative of the expanding and exciting role that proteomics-related technologies are now playing in the search for new ways of combating the formidable challenge to effective disease control that the malaria parasite still represents.

\section{Key issues}

- The proteome of Plasmodium falciparum, a single-celled eukaryotic parasite, is highly dynamic due to its complex life cycle in two disparate hosts and the numerous morphological and functional changes that it undergoes.

- This proteome is further complicated by the considerable degree of strain variation in $P$. falciparum populations.

- Proteome-wide studies have reported the identification of approximately half of the 5300 or so proteins predicted from the genome sequence of $P$. falciparum. Much has been done, but significant effort is still required to identify less abundant proteins.

- Some progress has been made in characterizing certain subpopulations of the proteome, an approach that will continue to be of value in tackling the proteomic complexity described above.

- Study of post-translational modifications is as important and complex in this parasite as in other organisms.

- Further development and wider use of negative-ion mass spectrometry methods are likely to make a significant contribution to future studies of both the unmodified proteome and its post-translational modifications.

- Adaptation of stable isotope labeling to parasite culture enables accurate quantification of changing protein levels in response to cell cycle progression and to perturbations such as drug inhibition and metabolic challenge.

- Only further quantitative studies will realize the full potential of parasite proteome analysis.

- The high proportion of proteins predicted from the genome sequence that are designated as hypothetical need to be verified at the proteomic level, as do bioinformatic predictions of subproteomes such as membrane transporters and secreted proteins. 
- Integration of transcriptomic, proteomic and, ultimately, metabolomic data is required for an in-depth understanding of parasite biology and rational drug design.

\section{Acknowledgments}

Relevant work performed in the authors' laboratories was funded principally by grant 073896 from the Wellcome Trust, UK.

\section{References}

Papers of special note have been highlighted as:

- of interest

•• of considerable interest

1. Pinswasdi C, Thaithong S, Beale GH, Fenton B, Webster HK, Pavanand K. Polymorphism of proteins in malaria parasites following mefloquine treatment. Mol. Biochem. Parasitol. 1987; 23(2): 159-164. [PubMed: 3553940]

2. Horgan G, Creasey A, Fenton B. Superimposing 2-dimensional gels to study genetic variation in malaria parasites. Electrophoresis. 1992; 13(11):871-875. [PubMed: 1483429]

3••. Gardner MJ, Hall N, Fung E, et al. Genome sequence of the human malaria parasite Plasmodium falciparum. Nature. 2002; 419(6906):498-511. Completion of the genome sequence was a major milestone and a prerequisite for the types of proteomic analysis described here. [PubMed: 12368864]

4••. Aebersold R, Mann M. Mass spectrometry-based proteomics. Nature. 2003; 422(6928):198-207. Very useful overview of instrumentation and methodologies that is accessible to the nonspecialist reader. [PubMed: 12634793]

5. Cohen AM, Rumpel K, Coombs GH, Wastling JM. Characterisation of global protein expression by two-dimensional electrophoresis and mass spectrometry: proteomics of Toxoplasma gondii. Int. J. Parasit. 2002; 32(1):39-51.

6••. Florens L, Washburn MP, Raine JD, et al. A proteomic view of the Plasmodium falciparum life cycle. Nature. 2002; 419(6906):520-526. Represents one of the first and most extensive analyses of the Plasmodium falciparum proteome to date. [PubMed: 12368866]

7••. Lasonder E, Ishihama Y, Andersen JS, et al. Analysis of the Plasmodium falciparum proteome by high-accuracy mass spectrometry. Nature. 2002; 419(6906):537-542. Represents one of the first and most extensive analyses of the $P$. falciparum proteome to date. [PubMed: 12368870]

8•. Hall N, Karras M, Raine JD, et al. A comprehensive survey of the Plasmodium life cycle by genomic, transcriptomic, and proteomic analyses. Science. 2005; 307(5706):82-86. An important attempt to integrate the various types of high-throughput data. [PubMed: 15637271]

9•. Sam-Yellowe TY, Florens L, Johnson JR, et al. A Plasmodium gene family encoding Maurer's cleft membrane proteins: structural properties and expression profiling. Genome Res. 2004; 14(6): 1052-1059. Excellent example of the power of subproteome analysis. [PubMed: 15140830]

10•. Vincensini L, Richert S, Blisnick T, et al. Proteomic analysis identifies novel proteins of the Maurer's clefts, a secretory compartment delivering Plasmodium falciparum proteins to the surface of its host cell. Mol. Cell. Proteomics. 2005; 4(4):582-593. Excellent example of the power of subproteome analysis. [PubMed: 15671043]

11. Nyalwidhe J, Baumeister S, Lingelbach K. Proteomic approach towards the characterization of the parasitophorous vacuole in Plasmodium falciparum-infected erythrocytes. Int. J. Med. Microbiol. 2004:29392-29393.

12. Kaneko O, Tsuboi T, Ling IT, et al. The high molecular mass rhoptry protein, RhopH1, is encoded by members of the clag multigene family in Plasmodium falciparum and Plasmodium yoelii. Mol. Biochem. Parasitol. 2001; 118(2):223-231. [PubMed: 11738712] 
13. Sam-Yellowe TY, Florens L, Wang TM, et al. Proteome analysis of rhoptry-enriched fractions isolated from Plasmodium merozoites. J. Proteome Res. 2004; 3(5):995-1001. [PubMed: 15473688]

14. Florens L, Liu X, Wang YF, et al. Proteomics approach reveals novel proteins on the surface of malaria-infected erythrocytes. Mol. Biochem. Parasitol. 2004; 135(1):1-11. [PubMed: 15287581]

15. Sinden RE. A proteomic analysis of malaria biology: integration of old literature and new technologies. Int. J. Parasit. 2004; 34(13-14):1441-1450.

16••. Khan SM, Franke-Fayard B, Mair GR, et al. Proteome analysis of separated male and female gametocytes reveals novel sex-specific Plasmodium biology. Cell. 2005; 121(5):675-687. Elegant combination of genetic engineering, cell-sorting and proteomic analysis to characterize a specific life-cycle stage. [PubMed: 15935755]

17. Campanale N, Nickel C, Daubenberger CA, et al. Identification and characterization of hemeinteracting proteins in the malaria parasite, Plasmodium falciparum. J. Biol. Chem. 2003; 278(30): 27354-27361. [PubMed: 12748176]

18. Knockaert M, Gray N, Damiens E, et al. Intracellular targets of cyclin-dependent kinase inhibitors: identification by affinity chromatography using immobilised inhibitors. Chem. Biol. 2000; 7(6): 411-422. [PubMed: 10873834]

19. Graves PR, Kwiek JJ, Fadden P, et al. Discovery of novel targets of quinoline drugs in the human purine binding proteome. Mol. Pharmacol. 2002; 62(6):1364-1372. [PubMed: 12435804]

20. Jeffery DA, Bogyo M. Chemical proteomics and its application to drug discovery. Drug Discov. Today. 2004; 9(2):S19-S26.

21. Doerig C, Meijer L, Mottram JC. Protein kinases as drug targets in parasitic protozoa. Trends Parasitol. 2002; 18(8):366-371. [PubMed: 12377287]

22. Phillips CI, Bogyo M. Proteomics meets microbiology: technical advances in the global mapping of protein expression and function. Cell. Microbiol. 2005; 7(8):1061-1076. [PubMed: 16008574]

23•. Fried M, Wendler JN, Mutabingwa TK, Duffy PE. Mass spectrometric analysis of Plasmodium falciparum erythrocyte membrane protein-1 variants expressed by placental malaria parasites. Proteomics. 2004; 4(4):1086-1093. Good example of the application of proteomics to the difficult area of surface-antigen variation. [PubMed: 15048989]

24. Salanti A, Dahlback M, Turner L, et al. Evidence for the involvement of VAR2CSA in pregnancyassociated malaria. J. Exp. Med. 2004; 200(9):1197-1203. [PubMed: 15520249]

25•. Sanders PR, Gilson PR, Cantin GT, et al. Distinct protein classes including novel merozoite surface antigens in raft-like membranes of Plasmodium falciparum. J. Biol. Chem. 2005; 280(48):40169-40176. A significant step in defining the key molecules of the merozoite surface. [PubMed: 16203726]

26••. Nirmalan N, Sims PFG, Hyde JE. Quantitative proteomics of the human malaria parasite Plasmodium falciparum and its application to studies of development and inhibition. Mol. Microbiol. 2004; 52(4):1187-1199. First and only method to date for fully quantitative proteomic analysis of $P$. falciparum. [PubMed: 15130134]

27. Gowda DC, Davidson EA. Protein glycosylation in the malaria parasite. Parasitol. Today. 1999; 15(4):147-152. [PubMed: 10322336]

28. Jones GL, Edmundson HM. Protein phosphorylation during the asexual life-cycle of the human malarial parasite Plasmodium falciparum. Biochim. Biophys. Acta. 1990; 1053(2-3):118-124. [PubMed: 2200523]

29. Suetterlin BW, Kappes B, Franklin RM. Localization and stage-specific phosphorylation of Plasmodium falciparum phosphoproteins during the intraerythrocytic cycle. Mol. Biochem. Parasitol. 1991; 46(1):113-122. [PubMed: 1852167]

30. Freitas-Junior LH, Hernandez-Rivas R, Ralph SA, et al. Telomeric heterochromatin propagation and histone acetylation control mutually exclusive expression of antigenic variation genes in malaria parasites. Cell. 2005; 121(1):25-36. [PubMed: 15820676]

31. Schmitz S, Grainger M, Howell S, et al. Malaria parasite actin filaments are very short. J. Mol. Biol. 2005; 349(1):113-125. [PubMed: 15876372] 
32. Gowda DC, Gupta P, Davidson EA. Glycosylphosphatidylinositol anchors represent the major carbohydrate modification in proteins of intraerythrocytic stage Plasmodium falciparum. J. Biol. Chem. 1997; 272(10):6428-6439. [PubMed: 9045667]

33. Gowda DC, Davidson E. More on protein glycosylation in the malaria parasite - Reply. Parasitol. Today. 2000; 16(1):39-40. [PubMed: 10637589]

34. Kimura EA, Katzin AM, Couto AS. More on protein glycosylation in the malaria parasite. Parasitol. Today. 2000; 16(1):38-39. [PubMed: 10637588]

35. Doerig C. Protein kinases as targets for antiparasitic chemotherapy. Biochim. Biophys. Acta Proteins Proteomics. 2004; 1697(1-2):155-168.

36•. Medzihradszky KF, Darula Z, Perlson E, et al. O-sulfonation of serine and threonine - mass spectrometric detection and characterization of a new posttranslational modification in diverse proteins throughout the eukaryotes. Mol. Cell. Proteomics. 2004; 3(5):429-440. Reports a previously unrecognised modification likely to be of functional significance in Plasmodium biology. [PubMed: 14752058]

37. Moore KL. The biology and enzymology of protein tyrosine O-sulfation. J. Biol. Chem. 2003; 278(27):24243-24246. [PubMed: 12730193]

38•. Gunasekera AM, Patankar S, Schug J, Eisen G, Wirth DF. Drug-induced alterations in gene expression of the asexual blood forms of Plasmodium falciparum. Mol. Microbiol. 2003; 50(4): 1229-1239. Investigates the effects of drug treatment on the transcriptome and proteome of $P$. falciparum. [PubMed: 14622411]

39•. Makanga M, Bray PG, Horrocks P, Ward SA. Towards a proteomic definition of CoArtem action in Plasmodium falciparum malaria. Proteomics. 2005; 5(7):1849-1858. Investigates the effects of drug treatment on the transcriptome and proteome of $P$. falciparum. [PubMed: 15832369]

40. Ong SE, Foster LJ, Mann M. Mass spectrometric-based approaches in quantitative proteomics. Methods. 2003; 29(2):124-130. [PubMed: 12606218]

41. Gygi SP, Rist B, Gerber SA, Turecek F, Gelb MH, Aebersold R. Quantitative analysis of complex protein mixtures using isotope-coded affinity tags. Nature Biotechnol. 1999; 17(10):994-999. [PubMed: 10504701]

42. DeSouza L, Diehl G, Rodrigues MJ, et al. Search for cancer markers from endometrial tissues using differentially labeled tags iTRAQ and clCAT with multidimensional liquid chromatography and tandem mass spectrometry. J. Proteome Res. 2005; 4(2):377-386. [PubMed: 15822913]

43. Smolka MB, Zhou HL, Purkayastha S, Aebersold R. Optimization of the isotope-coded affinity tag-labeling procedure for quantitative proteome analysis. Anal. Biochem. 2001; 297(1):25-31. [PubMed: 11567524]

44••. Ong S-E, Blagoev B, Kratchmarova I, et al. Stable isotope labeling by amino acids in cell culture, SILAC, as a simple and accurate approach to expression proteomics. Mol. Cell. Proteomics. 2002; 1(5):376-386. An important technological development from which the quantitative analysis of the $P$. falciparum proteome has been adapted. [PubMed: 12118079]

45. Bigwarfe PM, Wood TD. Effect of ionization mode in the analysis of proteolytic protein digests. Int. J. Mass Spectrom. 2004; 234(1-3):185-202.

46. Tholey A, Toll H, Huber CG. Separation and detection of phosphorylated and nonphosphorylated peptides in liquid chromatography-mass spectrometry using monolithic columns and acidic or alkaline mobile phases. Anal. Chem. 2005; 77(14):4618-4625. [PubMed: 16013881]

47. Syka JEP, Coon JJ, Schroeder MJ, Shabanowitz J, Hunt DF. Peptide and protein sequence analysis by electron transfer dissociation mass spectrometry. Proc. Natl Acad. Sci. USA. 2004; 101(26): 9528-9533. [PubMed: 15210983]

48. White NJ, Pongtavornpinyo W. The de novo selection of drug-resistant malaria parasites. Proc. R Soc. Lond. Ser. B-Biol. Sci. 2003; 270(1514):545-554.

49. Rathod PK, McErlean T, Lee PC. Variations in frequencies of drug resistance in Plasmodium falciparum. Proc. Natl Acad. Sci. USA. 1997; 94(17):9389-9393. [PubMed: 9256492]

50. Fairlamb AH. Metabolic pathway analysis in trypanosomes and malaria parasites. Philos. Trans. R Soc. Lond. Ser. B-Biol. Sci. 2002; 357(1417):101-107. [PubMed: 11839187]

51 • Le Roch KG, Johnson JR, Florens L, et al. Global analysis of transcript and protein levels across the Plasmodium falciparum life cycle. Genome Res. 2004; 14(11):2308-2318. An important 
analysis of the temporal relationship between the genome-wide levels of transcription and translation across parasite life-cycle stages. [PubMed: 15520293]

52. Martin RE, Henry RI, Abbey JL, Clements JD, Kirk K. The 'permeome' of the malaria parasite: an overview of the membrane transport proteins of Plasmodium falciparum. Genome Biol. 2005; 6(3):R26. Illustrates the contribution of bioinformatics analysis for the definition of important functionally related protein groupings. [PubMed: 15774027]

53. Hiller NL, Bhattacharjee S, van Ooij C, et al. A host-targeting signal in virulence proteins reveals a secretome in malarial infection. Science. 2004; 306(5703):1934-1937. Illustrates the contribution of bioinformatics analysis for the definition of important functionally related protein groupings. [PubMed: 15591203]

54•. Marti M, Good RT, Rug M, Knuepfer E, Cowman AF. Targeting malaria virulence and remodeling proteins to the host erythrocyte. Science. 2004; 306(5703):1930-1933. Illustrates the contribution of bioinformatics analysis for the definition of important functionally related protein groupings. [PubMed: 15591202]

55. Scholl PF, Kongkasuriyachai D, Demirev PA, et al. Rapid detection of malaria infection in vivo by laser desorption mass spectrometry. Am. J. Tropical Med. Hygiene. 2004; 71(5):546-551.

56. Marks F, Meyer CG, Sievertsen J, et al. Genotyping of Plasmodium falciparum pyrimethamine resistance by matrix-assisted laser desorption-ionization time-of-flight mass spectrometry. Antimicrob. Agents Chemother. 2004; 48(2):466-472. [PubMed: 14742196] 